\title{
Cdc42-mediated supracellular cytoskeleton induced cancer cell migration under low shear stress
}

Lingling Liua ${ }^{a}$, Hua Jiang ${ }^{b}$, Wei Zhao ${ }^{a}$, Yao Meng ${ }^{a}$, Ji Lic, Tongwei Huang ${ }^{d}$, JinghuiSuna

a. School of Laboratory Medicine, Collaborative Innovation Center of Sichuan for Elderly Care and Health, Development and Regeneration Key Laboratory of Sichuan Province, Sichuan Provincial Engineering Laboratory for Prevention and Control Technology of Veterinary Drug Residue in Animal-origin Food, Chengdu Medical College, Chengdu, 610500, Sichuan, China

b. Department of Pediatrics, Department of Microbiology and Immunology, Herman B. Wells Center for Pediatric Research, Indiana University School of Medicine, Indianapolis, IN, 46202, USA

c. Department of Clinical Laboratory, Sichuan GEM Flower Hospital, Chengdu, 610213, Sichuan, PR China

d. Department of Blood Transfusion, Ziyang Hospital of Traditional Chinese Medicine, Ziyang, 641300, Sichuan, PR China

This is the author's manuscript of the article published in final edited form as:

Liu, L., Jiang, H., Zhao, W., Meng, Y., Li, J., Huang, T., \& Sun, J. (2019). Cdc42-mediated supracellular cytoskeleton induced cancer cell migration under low shear stress. Biochemical and Biophysical Research Communications, 519(1), 134-140. https://doi.org/10.1016/j.bbrc.2019.08.149 


\section{HIGHLIGHTS}

- Low shear stress promotes needle-shaped structure formations.

- Activation of Cdc42 directly controls the filopodia characteristics.

- Needle-shaped protrusions contribute to the malignancy of tumor cells.

- Rac and Rho negatively affect the supracellular cytoskeleton structure.

\section{ABSTRACT}

Tumor microenvironment is composed of biological, chemical and physical factors. Mechanical factors are more and more focused these years. Therefore, mimicking mechanical factors' contribution to cancer cell malignancy will greatly improve the advance in this field. Although the induced malignant behaviors are present under many stimuli such as growth or inflammatory factors, the cell key physical migration mechanisms are still missing. In this study, we identify that low shear stress significantly promotes the formation of needle-shaped membrane protrusions, which is called filopodia and important for the sense and interact of a cell with extracellular matrix in the tumor microenvironment. Under low shear stress, the migration is promoted while it is inhibited in the presence of ROCK inhibitor Y27632, which could abolish the F-actin network. Using cell imaging, we further unravel that key to these protrusions is Cell division cycle 42 (Cdc42) dependent. After Cdc42 activation, the filopodia is more and longer, acting as massagers to pass the information from a cell to the microenvironment for its malignant phenotype. In the Cdc42 inhibition, the filopodia is greatly reduced. Moreover, small GTPases Cdc42 rather than Rac1 and Rho directly controls the filopodia formation. Our work highlights that low shear stress and Cdc42 activation are sufficient to promote filopodia formation, it not only points out the novel structure for cancer progression but also provides the experimental physical basis for the efficient drug anti-cancer strategies. 


\section{GRAPHICAL ABSTRACT}

0000000000000000000
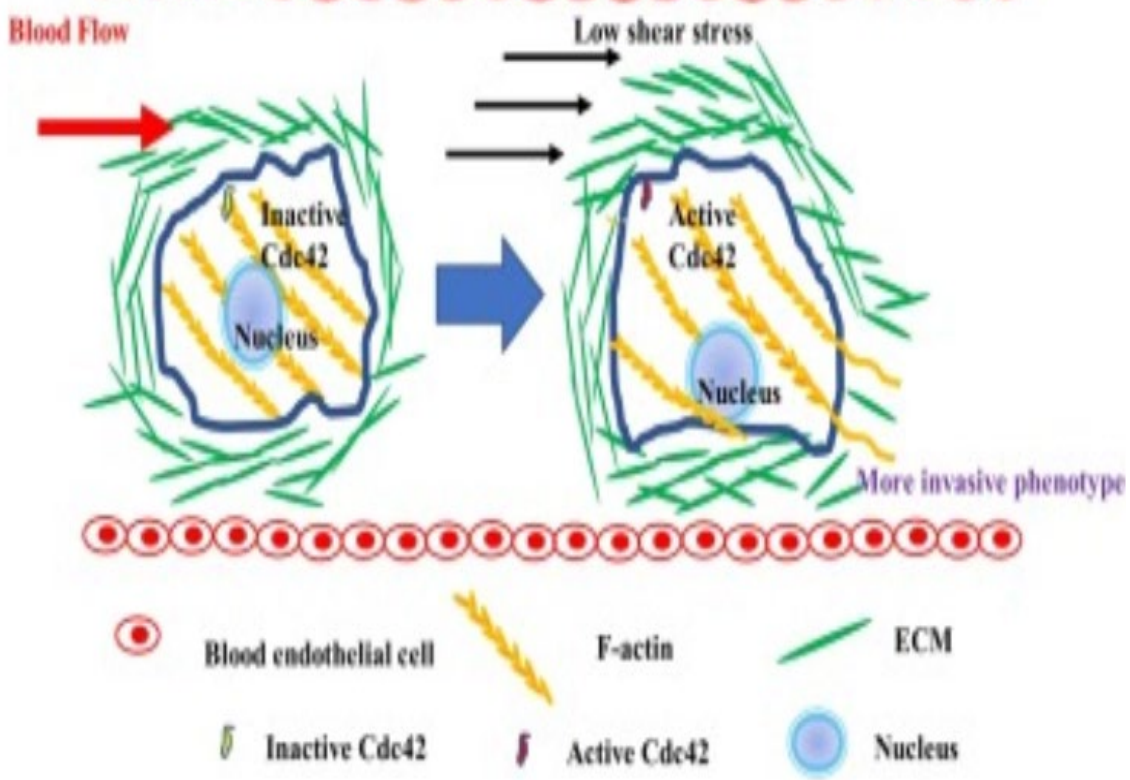

Keywords: Low shear stress, Filopodia, Migration, Mechanotransduction, Cdc42. 


\section{INTRODUCTION}

Bio-force is very important in developmental biology and physiology. In cardiovascular system, the main driving force of blood from heart to organ is biological force. Blood flow friction (low shear stress, LSS) can be perceived by cells and affect the biological characteristics of cells. Tumor cells are subjected to fluid shear stress when they invade blood vessels. This low shear stress can transform mechanical signals into biochemical signals, regulating gene expression and cell behavior [1,2]. Fluid shear stress is one of the important mechanical microenvironments in the process of distal metastasis of cancer cells. In and around normal or tumor tissues, shear stress alters the local mechanical microenvironment and regulates intracellular signaling pathways. In solid tumors, shear stress varies from $0.01 \mathrm{~Pa}$ to $0.2 \mathrm{~Pa}\left(0.1-2 \mathrm{dyne} / \mathrm{cm}^{2}\right)$ [3]. 2 dyne $/ \mathrm{cm}^{2}$ shear stress markedly upregulates matrix metalloproteinase-12 (MMP-12) expression and promotes chondrosarcoma cell invasion [4]. Previous study showed that low shear stress significantly promoted the proliferation of cancer cells [1], which showed the key role of mechanical factors in regulating cellular behaviors. Shah et al. proposed that the CXCR4/CXCL12 and MEK/ERK pathways were involved in the promotion of liver cancer cell migration in response to interstitial flow stimuli [5]. These studies fully show that biological forces have a significant effect on cells and can lead to different cell fates. It is necessary to explore the mechanism of low shear stress affecting the malignant evolution of cancer cells. Although the changes of cell response to microenvironment after stress stimulation are relatively clear, further research is needed on how cancer cells respond to shear stress and the effect of shear stress on cancer cell behavior and its molecular mechanism.

Needle-shaped protrusions (filopodia) are a kind of acicular cell membrane structure, which may have multiple roles. One is to connect cells and play a role of transferring molecules. The other is to participate in the interaction between cells and extracellular matrix as a mechanical receptor, participate in the movement of cells directly as pseudopodia. Needle-shaped structure can not only senses chemical signals in microenvironment, but also helps to sense physical signals in microenvironment. It is a key structure directly involved in the regulation of cancer cell motility in tumor 
microenvironment. Filopodia generally exists dynamically, participating in various biological processes, such as cell migration, wound closure, etc. [[6], [7], [8]]. This also indicates that filopodia plays a key role in the motility of tumor cells.

Filopodia perceives microenvironment, including chemical and physical signals. The most extensive study of chemical cue perception is the growth of cone guidance $[9,10]$. During wound healing, filopodia of epithelial cells are directly regulated by epidermal growth factor [11,12]. In addition to chemical cue sensing, filopodia can detect and sense mechanical signals of the physical environment, mainly through extracellular matrix exerting traction on the environment [13]. Therefore, filopodias are the direct manifestation of cell motility, but how they are formed and how they are related to the compliable and changeable extracellular microenvironment is still unknown.

Breast cancer develops through a complicated cascade involving tumorigenesis, increased motility, cell survival and colonization [14]. Rho GTPases serve as oncogenes in several human cancers, Rho family GTPases play a key role in cell migration and invasion by affecting cytoskeletal dynamics and turnover of adhesions $[15,16]$. The Rho family comprises a subfamily of the Ras superfamily of small GTPases, of which Rac, Rho and Cdc42 are included. Cdc42 is a small GTPase of the Rho family. Studies have shown that $\mathrm{Cdc} 42$ mediates the expression of integrin $\beta-1$, which promotes the interaction between cancer cells and endothelial cells and promotes the transmembrane movement of cancer cells [17]. Cdc42 has an inactive guanosine diphosphate (GDP)-bound state and an active GTP-bound active state, this is mediated by guanine-nucleotide-exchange factors (GEFs). The activation of Cdc42 could induce the signal pathway, thus the biological behaviors such as cytoskeletal changes, proliferation will be re-established. Hence, targeting Cdc42 represents a promising strategy for precise cancer therapy. As the initial step of malignant metastasis, the Cdc42 might be closely associated with the needle-shaped structure.

In this study, we chose to investigate whether LSS promotes breast cancer cell needleshaped structure formation and its regulatory mechanism. We demonstrate that LSS facilitates breast cancer cell needle-shaped structure formation as well as the migration ability. Cdc42 rather than Rac and Rho is involved in the filopodia formation which finally 
contributes to the cancer cell malignancy. These findings not only provide valuable evidence for cellular physical behaviors response to the microenvironment, but reveals a novel structure role of filopodia to initiate cancer cell malignant migration.

\section{MATERIALS AND METHODS}

\subsection{Reagents and antibodies}

Cell culture medium of L15, penicillin, streptomycin and fetal bovine serum (FBS) was purchased from Gibco (Grand Island, NY, USA). Phalloidin was purchased from SigmaAldrich (Saint Louis, MO, USA). Lipofectamine LTX were purchased from Thermo Fisher Scientific (Waltham, MA, USA), DAPI was purchased from Beyotime Biotechnology (Shanghai, CHINA). Y27632, ML141 and Rhosin were purchased from MedChemExpress (Monmouth Junction, NJ, USA). NSC was purchased from Selleck (Houston, TX, USA). 24-well Transwell plates were purchased from Becton Dickinson (Bedford, MA, USA). All other reagents were used as received without additional purification unless otherwise noted.

\subsection{Cell culture}

The triple negative human breast cancer cell MDA-MB-231 was obtained from the American Type Culture Collection (ATCC, Manassas, VA, USA). Cells were cultured in L15 medium composed of $10 \%$ fetal bovine serum and penicillin (100 U/mL) and streptomycin $(100 \mu \mathrm{g} / \mathrm{mL})$ at $37^{\circ} \mathrm{C}$. The culture media were replaced every 3 days. Confluent cells were trypsinized with $0.25 \%$ trypsin in $0.02 \%$ EDTA (Invitrogen, Carlsbad, USA) at $80 \%$ confluence and resuspended in the culture medium.

\subsection{Parallel flow chamber loading experiment}

Breast cancer line MDA-MB-231 were exposed to steady laminar low-level shear stress $\left(2 \mathrm{dyn} / \mathrm{cm}^{2}\right)$ using a parallel plated flow chamber system. The shear stress imposed to cells can vary from 0.5 to $37 \mathrm{dyn} / \mathrm{cm}^{2}$ through altering the flow rate. The flow chamber was connected to a recirculation flow loop, and a peristaltic pump (Masterflex, USA) was used to propel the culture medium from a reservoir. The culture medium was maintained 
at $37^{\circ} \mathrm{C}$ for $24 \mathrm{~h}$ throughout the experiment. Control cells were maintained under $37^{\circ} \mathrm{C}$ static conditions for the same length of time.

\subsection{Inhibition treatment}

MDA-MB-231 cells were treated with the selective inhibitor of ROCK (Y27632), which is an ATP-competitive inhibitor of ROCK-I and ROCK-II, selective inhibitor of Cdc42 (ML141), Rho inhibitor (Rhosin) and Rac inhibitor (NSC). Y27632 was at a concentration of $10 \mathrm{mM}^{*} 1 \mathrm{ml}$ in Dimethyl Sulphoxide (DMSO) as a stock solution (stored at $-20{ }^{\circ} \mathrm{C}$ ). The ML141, Rhosin and NSC stock solution was further diluted in cell culture medium to generate working concentrations, and DMSO was also used for all control. Prior to the LSS treatment, inhibitor was added to the medium 60 min with $10 \mu \mathrm{M}$ (Y27632), $100 \mu \mathrm{M}$ (ML141), $100 \mu \mathrm{M}$ (NSC), $30 \mu \mathrm{M}$ (Rhosin), respectively.

\subsection{Plasmids and transfection}

Plasmides encoding Cdc42-WT was a gift from Klaus Hahn (Addgene plasmid \# 12599), Cdc42-T17 N and Cdc42-Q61L were gifts from Gary Bokoch Addgene (plasmid \# 12976, Addgene plasmid \# 12986, respectively). Cells were trypsinized and quantified before plating on a glass. Cell density should be $50-80 \%$ confluent on the day of transfection. Cells were transfected with Lipofetamine LTX (Thermofisher, USA) according to the manufecturer's protocol. For each well, $0.5 \mu \mathrm{g}$ of DNA were diluted in $100 \mu \mathrm{L}$ of Opti$M E M \otimes$ reduced serum medium. For each well of cells, dilute $2 \mu$ l of Lipofectamine LTX into the diluted DNA solution, mix gently and incubate for $25 \mathrm{~min}$ at room temperature to form DNA-Lipofectamine LTX complexes. Incubate the cells at $37^{\circ} \mathrm{C}$ in humid incubator for 18-24 $\mathrm{h}$ post-transfection before assaying for transgene expression and F-actin distributions.

\subsection{Immunofluorescence staining}

The cells were washed with PBS and then fixed with 4\% paraformaldehyde in PBS for 15 min. After rinsing with PBS, the cells were permeabilized by treatment with $0.1 \%$ Triton X-100 in PBS for 30 min and rinsed with PBS, followed by incubation in 1\% BSA (bovine serum albumin) in PBS for $0.5 \mathrm{~h}$. Phalloidin was used according to the manufacturer's protocol. The nuclei were stained with DAPI for $5 \mathrm{~min}$. The fluorescence of the cells was 
imaged using a confocal laser scanning microscope (FV1000, Olympus, Japan). Optical density was used to measure the contents of specific cellular components. Finally, we used Image-Pro Plus graphical processing software to quantitatively analyze the length of filopodia.

\subsection{Cell migration and proliferation assay}

Cell migration was performed using transwell inserts (Millipore, Billerica, MA) with polycarbonate membranes of 8-mm pore size. Briefly, after exposure to shear stress loading, stretched or non-stretched cells (approximately $2 \times 10^{4}$ cells in $200 \mu \mathrm{L}$ medium without FBS) were added onto the insert and the insert was placed into a 24-well plate containing $700 \mu \mathrm{L}$ of medium supplemented with $1 \%$ FBS as a chemoattractant. After incubation for $6 \mathrm{~h}$ at $37^{\circ} \mathrm{C}$, the inner surfaces of the filters were swabbed to remove nonmigrating cells, and the outside cells were stained with $0.05 \%$ crystal violet in PBS for $30 \mathrm{~min}$. Cancer cells on the underside of the filters were counted under a microscope. Images were obtained from several randomly selected fields for each insert. Cell proliferation was determined using a cell counting kit-8 assay kit (CCK-8) (Dojindo, Kumamoto, Japan). Cells were reseeded in 96 -well plates at 5000 cells/well, cells were pretreated and then $20 \mu \mathrm{L}$ of CCK-8 solution was added to the medium, followed by further incubation for $1 \mathrm{~h}$. The absorbance at $450 \mathrm{~nm}$ was measured by spectrophotometry (BioTek, VT, USA).

\subsection{Statistical analysis}

Each experiment was repeated at least three times by the $t$-test for the detection of significant differences between the two groups. Data were collected as means \pm SDs. N.S. were considered to be no statistically significant difference, a value of $\left.p<0.001{ }^{* *}\right)$ were considered to be remarkably statistically significant.

\section{RESULTS}




\subsection{Low shear stress promoted breast cancer cell needle-shaped protrusions}

Needle-shaped protrusions are called filopodia, which is the sub-cellular structure acting as the messenger between the cell and the tumor microenvironment. DAPI can counterstain all nuclei and excite blue light. Fig. 1 showed that compared with the control group, the percentage of cells with filopodia under low shear stress (LSS)-treated group increased significantly, suggesting that LSS promoted the formation of filopodia in breast cancer cells. We also examined the filopodia length in all conditions, LSS-treated group was significantly longer than control. Meanwhile, ROCK inhibitor Y27632 significantly inhibited the percentage of cells with filopodia and filipodia length of MDA-MB-231 cells.
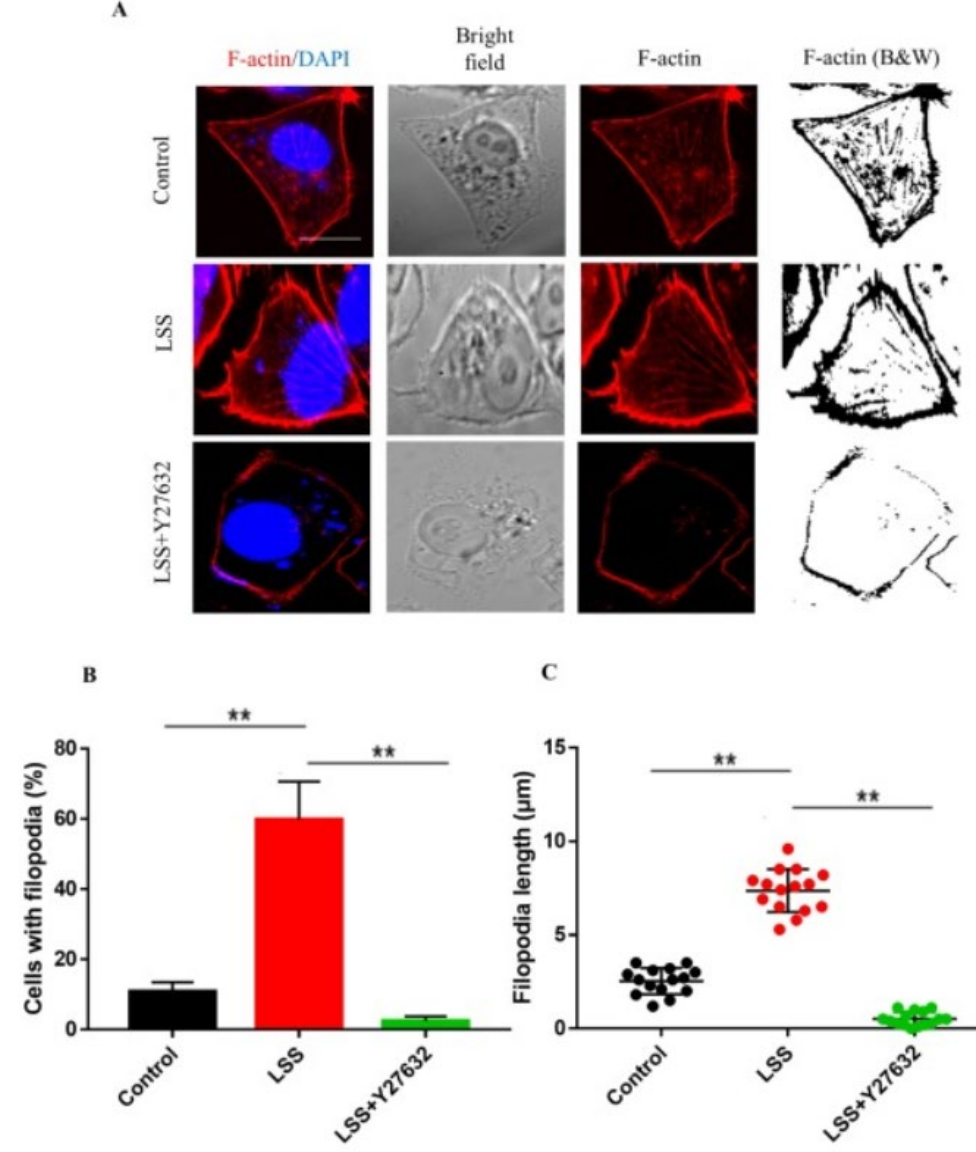

C

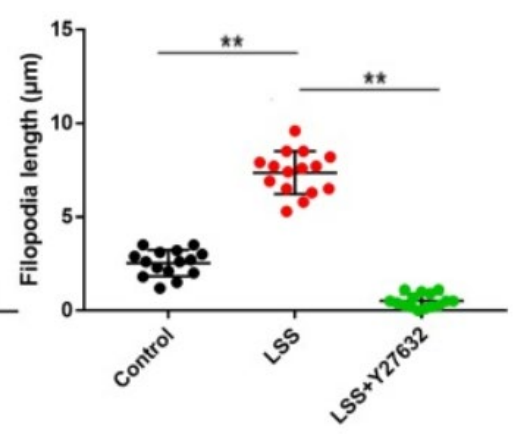

Fig. 1. Low shear stress promoted needle-shaped structure formation of MDA-MB231 breast cancer cell. (A) Immunofluorescence of F-actin (red) and DAPI (blue) at control, LSS and LSS with ROCK inhibitor Y27632 treatments (scale bar $=10 \mu \mathrm{m}$ ). (B) Quantitative percentage analysis of cells with filopodia. (C) Quantitative analysis of 
filopodia length in a cell, each dot is the average length of a cell. ${ }^{* *} p<0.001$ means significant difference.

\subsection{Low shear stress induced cancer cell migration}

To determine the relationship between filopodia and cell motility, transwell assay was used to detect breast cancer cell migration under LSS and LSS with Y27632 conditions. Compared with the control group, a significant difference of migration cells were observed between the control $(0 \mu \mathrm{M})$ and LSS-treated groups (Fig. 2a and b). The results showed that the number of LSS-treated cells that migrating to the lower chamber was significantly higher compared with the control cells. Meanwhile, the LSS with Y27632 group significantly decreased migrating cells, which indicated the role of filopodia in the migration process. Proliferation assay showed that there were no significant differences of the three groups during the migration hours, which excluded the potential effect that might be caused by cell proliferation (Fig. 2C).
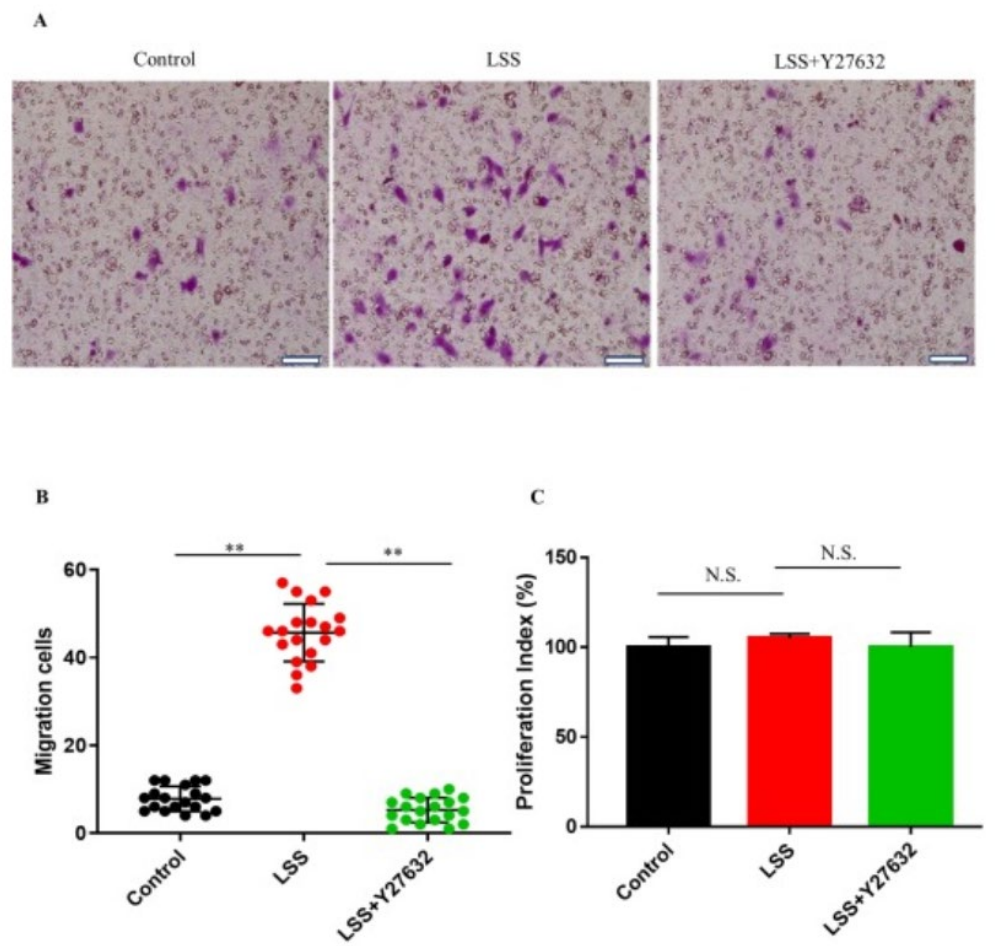

Fig. 2. Low shear stress induced MDA-MB-231 breast cancer cell migration. (A) Transwell migration assays showed that shear stress of $2 \mathrm{dyne} / \mathrm{cm}^{2}$ for $6 \mathrm{~h}$ promoted cell migration. Scale bar=100 mm. (B) Migration was quantified by counting cells on the lower 
face of each membrane, each dot was the average cell number in an image. (C) Proliferation assays showed the relative growth rates compare to control. Data are expressed as the mean $\pm S D$, N.S. means no significant difference while ${ }^{* *} p<0.001$ means significant difference.

\subsection{Cdc42 activation promoted filopodia formation}

We further examined filopodia formation upon different treatments. We used different expressions of Cdc42 mutants, Cdc42-WT, Cdc42-Q61L constitutively active and Cdc42 T17 $\mathrm{N}$ dominant negative. Fig. 3 showed that compare with control which the filopodia was not prominent, the expression of Cdc Q61L under no shear stress treatment group showed a significant increase to the percentage of cells with filipodia as well as filopodia length. Meanwhile, the expression of Cdc42T17 $\mathrm{N}$ failed to accumulate filopodia with cells and the filopodia length even with low shear stress, which suggested the Cdc42 was the main upstream molecule that controled the filopodia, and low shear stress was one stimulus that could accelerate the filopodia formation thus contributed to breast cancer cell motility. 


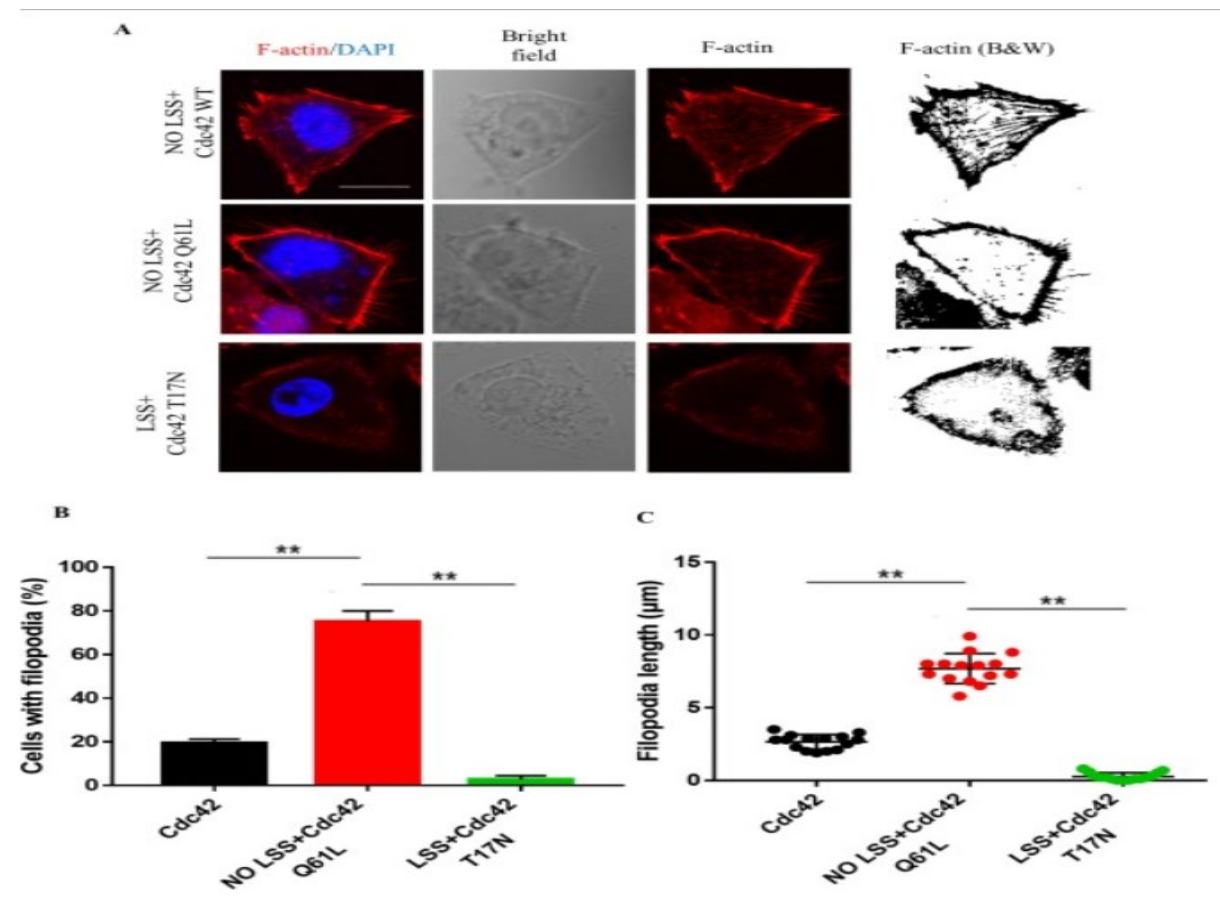

Fig. 3. Cdc42 activation directly controlled needle-shaped structure formation of MDA-MB-231 breast cancer cell. (A) Immunofluorescence of F-actin (red) and DAPI (blue) at expressing no LSS with Cdc42 wild type, no LSS with expression Cdc42 Q61L constitutively active mutant and LSS with Cdc42 T17 N dominant negative treatments (scale bar $=10 \mu \mathrm{m}$ ). (B) Quantitative percentage analysis of cells with filopodia. (C) Quantitative analysis of filopodia length in a cell, each dot was the average length of a cell. ${ }^{* *} p<0.001$ means significant difference.

\subsection{Cdc42 rather than Rac and Rho contributed to the supracellular cytoskeleton}

ML141 is a potent, selective and reversible non-competitive inhibitor of Cdc42 GTPase with low micromolar potency and selectivity against other members of the Rho family of GTPases (Rac1, Rab2, Rab7). NSC 23766 is an inhibitor of Rac GTPase, it targets Rac activation through guanine nucleotide factors (GEFs) and will not inhibit closely related targets, such as Cdc42 or RhoA. Rhosin is a potent and specific RhoA subfamily Rho GTPases inhibitor, which specifically binds to RhoA to inhibit RhoA-GEF interaction, and will not interact with Cdc42 or Rac1. We identified the role of different small GTPases in the supracellular cytoskeleton formation. Fig. 4 showed that compared with control (LSS group), Cdc42 inhibitor ML141 significantly reduced the cells with filopodia and the 
filopodia length, while Rac inhibitor NSC and Rho inhibitor Rhosin failed to decrease the filopodia. It suggested that Cdc42 rather than Rac and Rho controled the supracellular cytoskeleton structure formation, which revealed the different roles of the small GTPases during the cancer cell malignant progression.

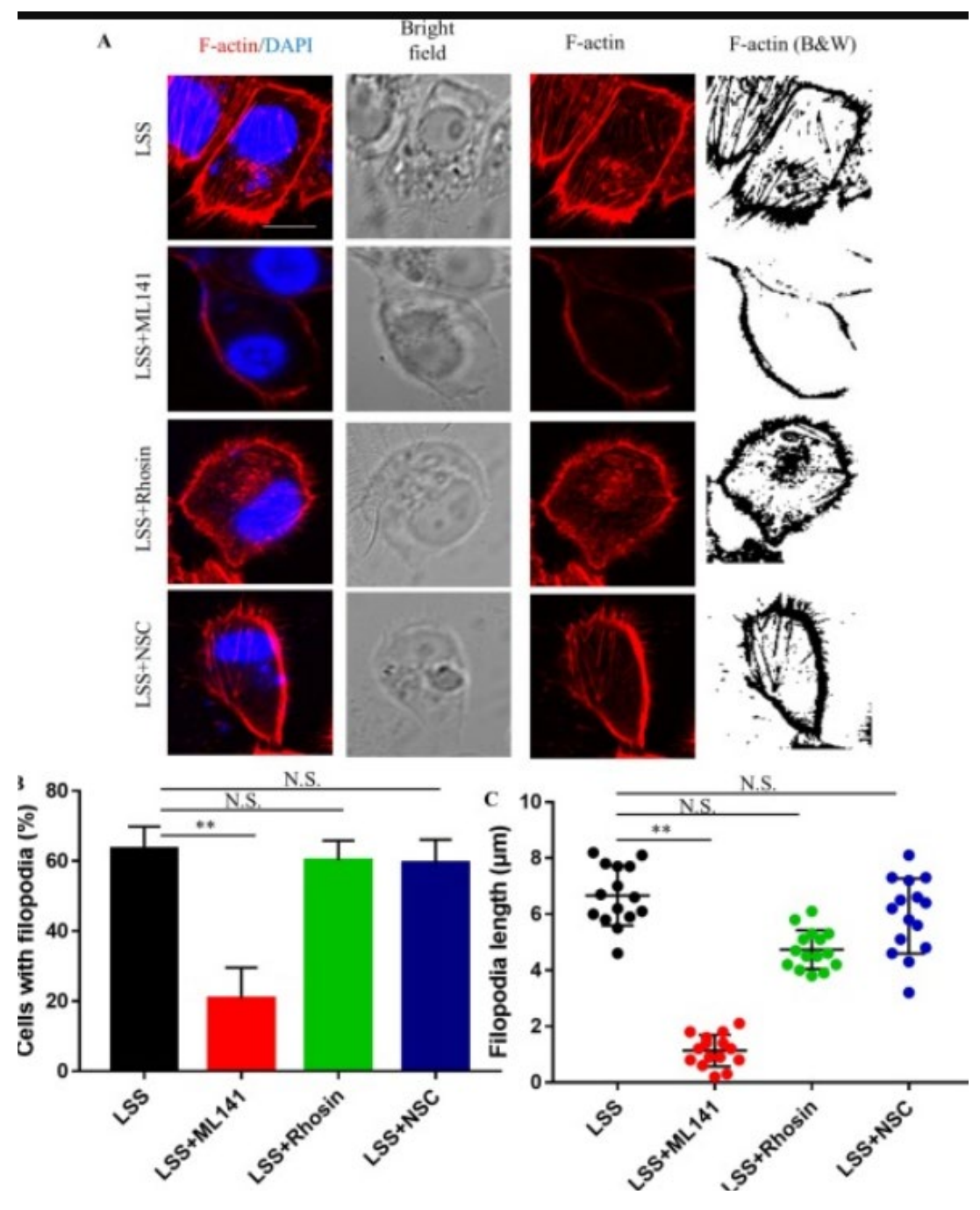

Fig. 4. Cdc42 activation rather than Rac and Rho directly controlled the supracellular cytoskeleton of MDA-MB-231 breast cancer cell. (A) Immunofluorescence of F-actin (red) and DAPI (blue) at expressing LSS, LSS with Cdc42 inhibitor ML141, Rho inhibitor Rhosin, Rac inhibitor NSC (scale bar $=10 \mu \mathrm{m}$ ). (B) Quantitative percentage analysis of cells with filopodia. (C) Quantitative analysis of filopodia length in a cell, each dot was the average length of a cell. ${ }^{* *} p<0.001$ means significant difference, N.S. means no significant difference. 
We conclude that LSS could promote breast cancer cell filopodia formation, which finally contributes to the cancer cell malignancy. The cell perceives the mechanical factors from the microenvironment, subsequently initiates a series of biological response, including the activation of $\mathrm{Cdc} 42$. Cdc42 directly controls the formation of needle-shaped protrusions, which leads the cells to be more malignant. The low shear stress is the cause of the malignancy and $\mathrm{Cdc} 42$ is the major receptor to receive the microenvironment, filopodia is the external display of cancer cell for its malignant progression.

\section{DISCUSSION}

Here, we have demonstrated that low shear stress governs the formation of filopodia and cancer cell migration ability. This supracellular network functions to initiate cell motility and $\mathrm{Cdc} 42$ is involved in this malignant metastasis. Therefore, it plays a critical role of mechanical tumor microenvironment to the cellular behaviors. Our finding provides a mechanism controlling filopodia formation which is closely associated with cancer cell motility.

Tumor microenvironment has a dynamic relationship with the development of cancer cell fate. To elucidate the molecular mechanism of tumor microenvironment in the process of influencing the fate of cancer cells is one of the key links to inhibit the malignant development of cancer. Biochemical factors play an important role in tumorigenesis and development. At the same time, more and more attention has been paid to mechanical factors $[18,19]$. There are many mechanical factors, such as shear stress and matrix stiffness, which are directly involved in the regulation of malignant behavior of cancer cells $[1,20]$. Tumor microenvironment affects tumor cells, which in turn remolds the tumor microenvironment. This study focused on the fluid shear stress in the process of malignant metastasis of tumors, explored the influence of this mechanical factor on the motility of tumors, and profoundly revealed the dynamic changes and promoting characteristics of shear stress in promoting the malignant evolution of tumors. Meanwhile, there are many factors affecting the tumor microenvironment. Shear stress may also affect the malignant evolution of tumors by synergistic action with chemical factors such as chemokine or growth factor and mechanical factors such as matrix stiffness. The 
mechanism of synergistic regulation still needs further study to reveal the effect of complex tumor microenvironment.

Rho family GTPases actively participates in cancer cell malignancy, affecting the F-actin polymerization. The family comprise a subfamily of the Ras superfamily of small GTPases, of which Rac, Rho and Cdc42, and they are all related to cell adhesion, which indicates the potential controlling roles for cell motility [21]. When a cell starts the malignant metastasis, they are mostly stimulated by factors in tumor microenvironment. The cells need a structure which could not only connect the cell with the extracellular microenvironment, but also crosstalk with the neighboring cells to achieve coordinated regulation. In this process, the filopodia in our study showed the potential to meet the demand. The needle-shaped structure could directly sense the factors in the microenvironment and it could also act as a messenger to pass the information from one cell to another. Our findings highlight the mechano-sensory role of filopodia, which directly display as the cells with filopodia and filopodia length, unraveling a novel structure during efficient metastasis. Therefore, the structure is important to initiate and accelerate cancer cell malignant evolution.

How does Cdc42 govern filopodia? Before our studies, the study between extracellular matrix and cell adhesion have been known to mainly control the formation and patterns of basal stress fibers during morphogenesis [22]. Meanwhile, in breast cancer proliferation, Cdc42 functions as an EGFR-signaling regulator, that EGFR and Cdc42 positive loop is necessary to activate Cdc42 $[23,24]$. However, the correlation and link between adhesion complex and Cdc42 activity are unclear. The further answer to this question will improve our understanding of the filopodia in the future. In our study, Cdc 42 overexpression leads to more filopodia formation, thus provide a structure to contract the cell, detach the rear and move the cells forward. It is the whole story to reveal the sprout of metastasis, the cells perceive the mechanical stimuli from low shear stress in tumor microenvironment, via Cdc42 to promote the formation of filopodia in cells and percentage of cells with filopodia, ready for the malignant metastasis. The story reveals the important process during the tumor evolution and it is cross validated with the biological behaviors in different tumor microenvironment. Cdc42 also might have correlation with microtubule 
or cell adhesions, revealing the role of cdc42 in malignancy will greatly improve our standing to anti-cancer strategy. Targeting Cdc42 provides a promising strategy for precise breast cancer therapy or prevention.

In summary, we observed an LSS-induced cancer cell needle-shaped structure formation. LSS significantly promotes the percentage of cells with filopodia and filopodia length, which are closely associated with cancer cell motility. Further, Cdc42 is directly involved in the filopodia formation, which indicates that Cdc42 directly participates in the lowshear-stress-stimulated migration in the signal transmissions. In addition, the observed filopodia structure suggests the mechanical factors can be converted to cellular physical behaviors for the cancer cell malignancy, thus accelerating cancer progression.

\section{Conflicts of interest}

The authors declare that there are no conflicts of interest.

\section{ACKNOWLEDGEMENTS}

This work was supported, in part or in whole, by the grants from Health Department of Sichuan Province (18PJ002) and the Key Project of Sichuan Education Department (18ZA0141). The General Program of School of Laboratory Medicine (JYZK201601, JYZK201702), Chengdu Medical College (CYZ17-20, CYZ1613). The Research Fund of Development and Regeneration Key Laboratory of Sichuan Province (SYS16-009, SYS18-08). Technology Innovation R\&D Project of Chengdu Science and Technology Bureau (2018-YFYF-00158-SN). Collaborative Innovation Center of Sichuan for Elderly Care and Health (YLZBZ1802). 


\section{REFERENCES}

1. Qin, X., Li, J., Sun, J., Liu, L., Chen, D., \& Liu, Y. (2019). Low shear stress induces ERK nuclear localization and YAP activation to control the proliferation of breast cancer cells. Biochemical and Biophysical Research Communications, 510(2), 219-223. https://doi.org/10.1016/j.bbrc.2019.01.065

2. Sun, J., Luo, Q., Liu, L., \& Song, G. (2019). Low-level shear stress induces differentiation of liver cancer stem cells via the $W n t / \beta$-catenin signalling pathway. Experimental Cell Research, 375(1), 90-96. https://doi.org/10.1016/j.yexcr.2018.12.023

3. Polacheck, W. J., Charest, J. L., \& Kamm, R. D. (2011). Interstitial flow influences direction of tumor cell migration through competing mechanisms. Proceedings of the National Academy of Sciences, 108(27), 11115-11120. https://doi.org/10.1073/pnas.1103581108

4. Wang, P., Chen, S.-H., Hung, W.-C., Paul, C., Zhu, F., Guan, P.-P., Huso, D. L., Kontrogianni-Konstantopoulos, A., \& Konstantopoulos, K. (2015). Fluid shear promotes chondrosarcoma cell invasion by activating matrix metalloproteinase 12 via IGF-2 and VEGF signaling pathways. Oncogene, 34(35), 4558-4569. https://doi.org/10.1038/onc.2014.397

5. Shah, A. D., Bouchard, M. J., \& Shieh, A. C. (2015). Interstitial Fluid Flow Increases Hepatocellular Carcinoma Cell Invasion through CXCR4/CXCL12 and MEK/ERK Signaling. PLOS ONE, 10(11), e0142337. https://doi.org/10.1371/journal.pone.0142337

6. Mattila, P. K., \& Lappalainen, P. (2008). Filopodia: Molecular architecture and cellular functions. Nature Reviews Molecular Cell Biology, 9(6), 446-454. https://doi.org/10.1038/nrm2406

7. Mellor, H. (2010). The role of formins in filopodia formation. Biochimica et Biophysica Acta (BBA) - Molecular Cell Research, 1803(2), 191-200. https://doi.org/10.1016/j.bbamcr.2008.12.018

8. Faix, J., Breitsprecher, D., Stradal, T. E. B., \& Rottner, K. (2009). Filopodia: Complex models for simple rods. The International Journal of Biochemistry \& Cell Biology, 41(8), 1656-1664. https://doi.org/10.1016/j.biocel.2009.02.012 
9. Gallo, G., \& Letourneau, P. C. (2004). Regulation of growth cone actin filaments by guidance cues. Journal of Neurobiology, 58(1), 92-102. https://doi.org/10.1002/neu.10282

10. Geraldo, S., \& Gordon-Weeks, P. R. (2009). Cytoskeletal dynamics in growth-cone steering. Journal of Cell Science, 122(20), 3595-3604. https://doi.org/10.1242/jcs.042309

11. Lidke, D. S., Lidke, K. A., Rieger, B., Jovin, T. M., \& Arndt-Jovin, D. J. (2005). Reaching out for signals filopodia sense EGF and respond by directed retrograde transport of activated receptors. Journal of Cell Biology, 170(4), 619-626. https://doi.org/10.1083/jcb.200503140

12. Vasioukhin, V., Bauer, C., Yin, M., \& Fuchs, E. (2000). Directed Actin Polymerization Is the Driving Force for Epithelial Cell-Cell Adhesion. Cell, 100(2), 209-219. https://doi.org/10.1016/S0092-8674(00)81559-7

13. Chan, C. E., \& Odde, D. J. (2008). Traction Dynamics of Filopodia on Compliant Substrates. Science, 322(5908), 1687-1691. https://doi.org/10.1126/science.1163595

14.Zhang, Y., Li, J., Lai, X.-N., Jiao, X.-Q., Xiong, J.-P., \& Xiong, L.-X. (2019). Focus on Cdc42 in Breast Cancer: New Insights, Target Therapy Development and Non-Coding RNAs. Cells, 8(2), 146. https://doi.org/10.3390/cells8020146

15. Vega, F. M., \& Ridley, A. J. (2008). Rho GTPases in cancer cell biology. FEBS Letters, 582(14), 2093-2101. https://doi.org/10.1016/j.febslet.2008.04.039

16. Ridley, A. J. (2011). Life at the Leading Edge. Cell, 145(7), 1012-1022. https://doi.org/10.1016/j.cell.2011.06.010

17. Reymond, N., Im, J. H., Garg, R., Vega, F. M., Borda d'Agua, B., Riou, P., Cox, S., Valderrama, F., Muschel, R. J., \& Ridley, A. J. (2012). Cdc42 promotes transendothelial migration of cancer cells through $\beta 1$ integrin. Journal of Cell Biology, 199(4), 653-668. https://doi.org/10.1083/jcb.201205169

18. You, Y., Zheng, Q., Dong, Y., Xie, X., Wang, Y., Wu, S., Zhang, L., Wang, Y., Xue, T., Wang, Z., Chen, R., Wang, Y., Cui, J., \& Ren, Z. (2016). Matrix stiffness-mediated effects on stemness characteristics occurring in HCC cells. Oncotarget, 7(22), 32221-32231. https://doi.org/10.18632/oncotarget.8515 
19. Mollica Poeta, V., Massara, M., Capucetti, A., \& Bonecchi, R. (2019). Chemokines and Chemokine Receptors: New Targets for Cancer Immunotherapy. Frontiers in Immunology, 10. https://doi.org/10.3389/fimmu.2019.00379

20.Page, J. M., Merkel, A. R., Ruppender, N. S., Guo, R., Dadwal, U. C., Cannonier, S. A., Basu, S., Guelcher, S. A., \& Sterling, J. A. (2015). Matrix rigidity regulates the transition of tumor cells to a bone-destructive phenotype through integrin $\beta 3$ and TGF- $\beta$ receptor type II. Biomaterials, 64, 33-44. https://doi.org/10.1016/j.biomaterials.2015.06.026

21. Aspenström, P. (2020). Fast-cycling Rho GTPases. Small GTPases, 11(4), 248-255. https://doi.org/10.1080/21541248.2017.1391365

22. Haigo, S. L., \& Bilder, D. (2011). Global Tissue Revolutions in a Morphogenetic Movement Controlling Elongation. Science, 331(6020), 1071-1074. https://doi.org/10.1126/science.1199424

23. Hirsch, D. S., Shen, Y., \& Wu, W. J. (2006). Growth and Motility Inhibition of Breast Cancer Cells by Epidermal Growth Factor Receptor Degradation Is Correlated with Inactivation of Cdc42. Cancer Research, 66(7), 3523-3530. https://doi.org/10.1158/00085472.CAN-05-1547

24. Tu, S., \& Cerione, R. A. (2001). Cdc42 Is a Substrate for Caspases and Influences Fasinduced Apoptosis. Journal of Biological Chemistry, 276(22), 19656-19663. https://doi.org/10.1074/jbc.M009838200 